\title{
A Systematic Framework of Equipment Maintenance and Service with Application to Wire Bonder
}

\author{
Ren-Jung Chang ${ }^{1}$, Yu-Tsang Hsieh ${ }^{2}$, Eric Chang ${ }^{3}$ \\ ${ }^{1}$ Department of Mechanical Engineering, National Cheng Kung University, Taiwan, China; ${ }^{2}$ Customer Operations, Kulicke and Soffa \\ Asiapac Inc., Taiwan, China; ${ }^{3}$ Narmo International Inc., Alberta, Canada. \\ Email: rjchang@mail.ncku.edu.tw, ahsieh@kns.com, eric@narmo.com
}

Received August 11 $1^{\text {th }}, 2009$; revised October $2^{\text {nd }}, 2009$; accepted November $17^{\text {th }}, 2009$.

\begin{abstract}
A systematic framework for the maintenance and service of equipment is developed and proposed. The framework consists of a system of equipment failure analysis, methods, process, and activities, and a procedure in maintenance and service. With axiomatic design mapping, the maintenance procedure is constructed by integrating value engineering, quality function deployment, mechatronics engineering, technique from $R \& D$ and supplier, and Taguchi method. The maintenance and service of a wire bonder machine, K\&S (Kulicke and Soffa) Maxum Plus, in the first bond failure is employed for illustration.
\end{abstract}

Keywords: Systematic Framework, Maintenance, Service, Wire Bonder, Failure Modes, Mechatronics, Axiomatic Design, Taguchi Method

\section{Introduction}

Since the first development of ball bonding technique by the Bell Laboratories, more than five trillion wires have been bonded onto various semiconductor devices routinely every year [1]. Today, with the increasing equipment and workload in a semiconductor business, customers are relying on suppliers to provide efficient and effective maintenance and service. The importance of maintenance function and its management has grown rapidly. As a matter of fact, some research efforts have been undertaken on the maintenance related issues in the semiconductor business through out the years $[2,3]$. For example, integration of failure analysis in microelectronics manufacturing process was proposed for cost reduction [2]. In the research area, many research efforts have been undertaken on the field of maintenance management. The objectives cover the fields ranging from optimization models, maintenance techniques, scheduling, performance measurement, information systems to policies [4]. In the literature review [4], most of the researches have been established on developing general methods for the development and operation of maintenance system. However, methods for the maintenance and service of equipment in the troubleshooting of system failure and assuring production quality to satisfy customer's needs are seldom published.

Maintenance engineering and service operation allow customers to have on-going and routine access with experienced engineering experts for minimizing the effort of maintenance management. In addition, it allows customers to focus on their core competencies and areas of individual specialty. Maintenance is an engineering decision and associated actions for the optimization of specified capabilities [5]. A service is to perform an action for customer to keep a product in good operating condition. The benefits of maintenance procedures in manufacturing environment will not only optimize machine reliability to reduce production costs but also improve product quality to increase yield. The service operation enhances the technical oversight of specialized engineering operations at a fraction of the required cost to maintain the performance in-house. Development of a systematic engineering method for efficient and effective maintenance and service of semiconductor equipment should provide large returns in productivity for a semiconductor fabrication business.

In this paper, a novel framework is proposed and applied for the maintenance and service of semiconductor equipment. A systematic framework of equipment maintenance and service is first proposed. Next, a mechatronics structure integrated with axiomatic design mapping of a wire bonder is constructed for documenting the know- 
ledge of equipment. Then, the framework is employed for troubleshooting the first bond failure and maintenance service. Finally, the contribution by the present approach on the maintenance and service of equipment is concluded.

\section{Systematic Framework of Maintenance and Service}

Maintenance activities in engineering service will increase machine utilization and improve productivity. There are several activities such as Failure Mode and Effects Analysis (FMEA), troubleshooting, and process optimization involved in the maintenance and service of equipment. In this section, a systematic framework is developed for providing an efficient and effective maintenance and service.

\subsection{Engineering System of Failure Analysis}

Engineering maintenance and service usually involve a complex combination of resources. A system engineering method is required to manage engineering resources to fulfill customer's needs [6]. For providing maintenance service to customers, qualified engineers are to be trained through laboratory and field practice. Engineer training usually includes practicing technical skill, writing reports, and studying ethics. Engineering measuring and testing techniques regarding semiconductor equipment are required and built on the knowledge among a production field. All related information in maintenance practice is to be documented to improve engineer skill and attitude. In failure diagnosis, failure criteria are required a priori to classify operating status. For providing an optimal solution in reducing the failure probability over time, the analysis of the value in industrial environment for the operation of equipments will be tracked and controlled for every aspect of routine and non-routine equipment maintenance.

For the system involved in failure analysis and maintenance, the objective is to optimize maintenance function and servicing cost to satisfy customer's needs. For system engineering management, the system environment and components are to be identified. The activities of equipment failure analysis and maintenance are deemed to be operated in an industrial environment. The industrial environment may include human and material resources, industrial standards and technologies, political, economic and social factors, and international involvement [6]. For the system components involved in the activities, five components can be identified as Maintenance/Service Engineer, Machine/System Knowledge, Measurement and its Knowledge, Failure Criteria, and Maintenance Documentation. In order to operate maintenance system, all activities are to be relied on maintenance/service engineer. Therefore, the component of
Maintenance/Service Engineer will interact with all the other four components. From the above analysis, a system model of equipment failure analysis and maintenance for engineering service is constructed and proposed as depicted in Figure 1.

\subsection{Methods, Process, and Activities}

A scheme to integrate various methods in maintenance process is constructed for machine diagnostics and process optimization of semiconductor equipment. Several related techniques and methods to be integrated for realizing the system of equipment failure analysis and maintenance in Figure 1 will be briefly introduced.

Value engineering provides a measure of value for the purpose of maintenance and service of semiconductor equipment. Value can be defined as function over cost [6]. The value of maintenance and service for different failure modes needs to be evaluated. The ultimate goal of the maintenance and service support in the fabrication industries is to increase the net value by reducing the cost, and increasing customer's acceptance during the equipment's life cycle.

The conversion from customer's needs to functional requirements in equipment development is an essential issue in a semiconductor business. A systematic method of Quality Function Deployment (QFD) can be applied quantitatively to solve the conversion problem. In realizing QFD, a central element called House of Quality is usually employed. The success of QFD relies on the detailed investigation of the customer's needs and the evaluation of the markets. The initial tasks in QFD are to acquire market needs by listening to the voice of customer, sorting the needs, and prioritizing the needs numerically. In reliability engineering, the attempt of introducing an integrated usage of QFD and FMEA has been undertaken by several researchers [7].

A machine as a system can be decomposed into many sub-systems for engineering operation and testing. In the

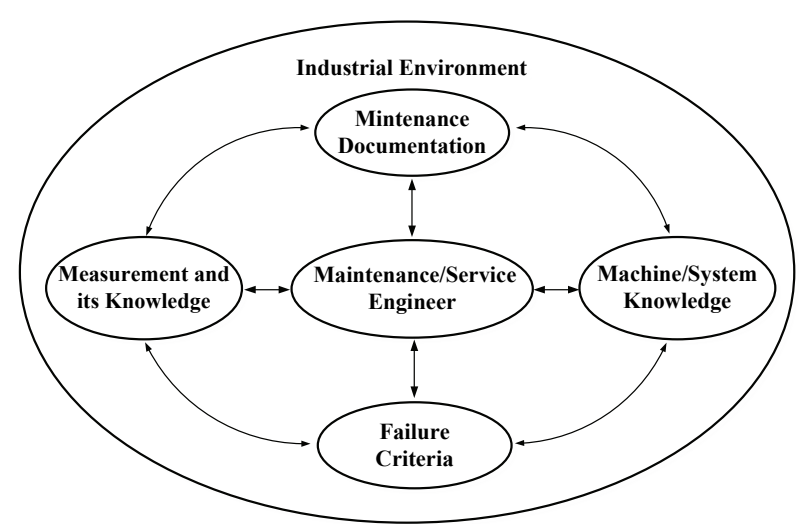

Figure 1. Engineering system of failure analysis in maintenance and service 
decomposition of semiconductor equipment, it is essential to understand the equipment's principle and mechanism in operation. The design and implementation of the semiconductor equipment relies on the integration of precision mechanical engineering, electrical control, and system thinking. According to the definition of mechatronics by EEC [8], the technique and the knowledge of semiconductor equipment is classified as a discipline of mechatronics. With the knowledge of mechatronics in semiconductor equipment, failure analysis and maintenance can be undertaken by engineers in service.

Semiconductor equipment is designed and implemented through precision engineering. In the field of precision design, Suh [9] identified two design axioms from abstracting common mapping domains of a body of precision designs, including products, processes, and systems. The foundation of axiomatic design is to construct design mappings in the forward engineering. The design mappings are from Customer Attributes (CAs) to Functional Requirements (FRs), then from FRs to Design Parameters (DPs) and finally from DPs to Process Variables (PVs). Recently, the design method has been integrated with other methods for different applications. For example, a design method to integrate the axiomatic design and robust design was developed for assuring six-sigma quality in product [10]. In addition, some thoughts were proposed on potential integration of axiomatic design with quality tools to enhance product development process [11].

For a semiconductor fabrication process, minimizing the process variations is the fundamental objective in achieving the optimal machine operation. There has long been a desire to increase the yield by utilizing an electrical or mechanical in-process quality control system. Engineers working on designing quality into products and processes should be provided with a tool available for the experimental design in the field tests. Taguchi has developed a robust method for improving the quality of Japanese products with great success [12]. Taguchi's method is a robust method for improving productivity so that high-quality products can be produced at low cost
[13]. The robust method, in essence, is to utilize a Signal-to-Noise $(\mathrm{S} / \mathrm{N})$ ratio to estimate and assure the minimum variability in product quality.

Maintenance engineers need to have full knowledge about machine failure in maintenance service. In fabrication industry, a method of FMEA provides a structured approach to analyze the root causes of failure, and assesses the potential failure modes of a process and their causes in risk prevention measures $[6,14]$. In utilizing FMEA, an integral part is to calculate Risk Priority Number (RPN) for ranking and assessing risk of failure modes [14]. The RPN is used to prioritize items of causes that require additional quality planning or action. The items with the highest RPN and severity ratings usually will be given first consideration. However, the priorities ranking by RPN may not always reflect the true concerns in maintenance management [15].

In the operation of semiconductor equipment, the operational process and equipment setting in production are usually relied on the techniques from R\&D and supplier. The operational techniques usually are to be trained through laboratory and field practice.

In the applications of precision engineering to the semiconductor industry, there should also be an efficient and effective maintenance system for providing high performance and quality operations at a reasonable cost. In considering that axiomatic design can be used as the foundation in designing semiconductor equipment, a systematic method through axiomatic design mapping is proposed for engineers to solve the maintenance problems. By integrating the engineering methods mentioned in the previous paragraphs along with the forward and inverse mappings in axiomatic design, a scheme to integrate various methods in maintenance process for machine diagnostics and process optimization of semiconductor equipment is constructed and depicted in Figure 2.

In Figure 2, the maintenance needs are initially raised by the customer. In proceeding maintenance process, the value and worth of maintenance will be analyzed and evaluated. The value analysis requires the mapping between the CAs and FRs. The FRs can be deployed and

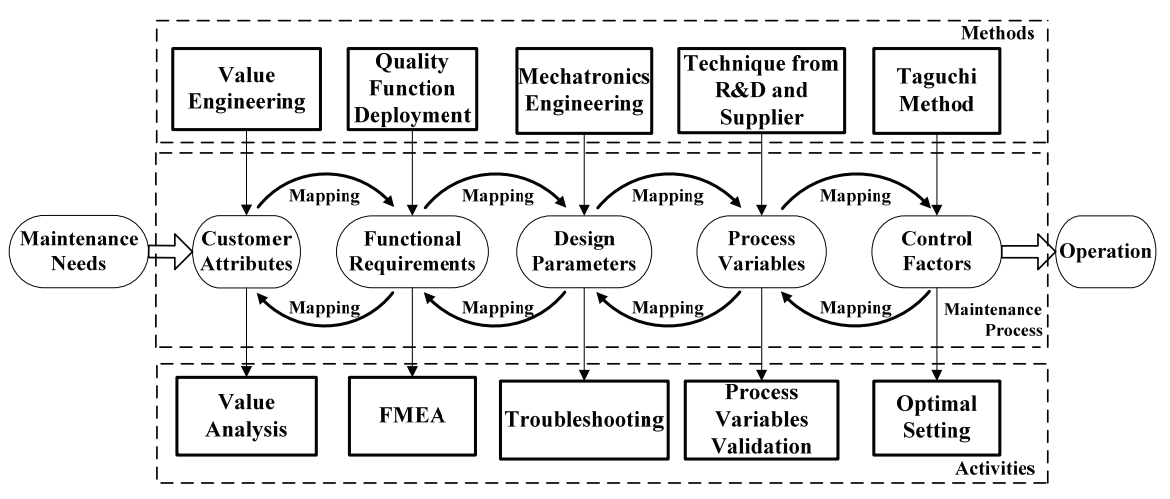

Figure 2. Methods, process, and activities in maintenance and service 
defined based on the analysis of quality function. By analyzing and evaluating the maintenance needs and FRs, CAs are determined by using the method of value engineering. In proceeding the analysis of FRs in maintenance, FMEA is undertaken to obtain causes and effects of failure modes. For semiconductor equipment, various components are mutually affected regarding the behaviors of machine and its performance. By utilizing mechatronic engineering, the forward mapping from the FRs to DPs is analyzed to give the reverse mapping of DPs that affect the FRs. With the mapping from the FRs to DPs, the DPs which affect FRs can be verified in troubleshooting during maintenance. With the DPs, a mapping from the DPs to PVs for semiconductor equipment can be constructed. The PVs will affect the variations of DPs in equipment during the life cycle of equipment operation. For the PVs, some PVs related to fabrication processes which are sensitive to disturbances will be involved in quality problems. By assuming a set of PVs which may affect the quality specifications, laboratory experiments can be carried out by supplier or through R\&D for identifying the key PVs. The key PVs which are defined as Control Factors (CFs) are employed for assuring production quality. In production, an important aspect of quality in setting CFs is to achieve robustness. Since the issue of robustness cannot be addressed until noise factors are given in an environment, designed experiments by Taguchi method are utilized to find out the optimal setting of CFs for minimizing product variance during equipment operation.

\subsection{Procedure}

Under the diagram constructed in Figure 2, a procedure for realizing maintenance and service will be developed. In the maintenance procedure starting from Maintenance Needs to Operation, there are five activities associated with the mapping domains, respectively. The five activities including Value Analysis, FMEA, Troubleshooting, Process Variables Validation, and Optimal Setting will proceed with the following steps.

1) Value Analysis

At first, maintenance needs are initiated by the customer. Therefore, from the initiation of maintenance needs, CAs will be reviewed and revised and the value in CAs will be evaluated iteratively. If the maintenance service is valuable for proceeding the troubleshooting and/or process optimization, the FMEA will be undertaken to analyze the root causes of failure modes.

\section{2) FMEA}

In proceeding with the FMEA, the failure problem will be defined in operation environment. In addition, information of potential effects of failure and risk factor rating are analyzed and documented. The analysis of failure modes and their causes can be inferred by identifying the failure problem, reviewing FRs, and the corresponding
DPs iteratively.

3) Troubleshooting

After FMEA, field tests are undertaken for troubleshooting. The failure due to mechanism or system software in semiconductor equipment will be tested first. The troubleshooting is undertaken by iterating test and check in order to resolve any issues of DPs in the subsystems or components.

4) Process Variables Validation

If the equipment is functionally correct, the equipment will be controlled and tested by setting PVs in fabrication process software. The operation of equipment will be validated iteratively by reviewing and revising PVs to make sure that PVs are correct and no failures occur in the fabrication process software.

5) Optimal Setting

If the equipment operates correctly under the control of fabrication process software, then experimental tests will be applied for process optimization. By analyzing the CFs and noise factors in PVs and testing the CFs in fabrication process software, Taguchi method is applied through reviewing CFs iteratively for obtaining optimal $\mathrm{CFs}$.

The maintenance and service procedure is completed after setting the optimal CFs. The implementation of the maintenance and service procedure will indicate that the failure has been resolved and the machine performance is robust in operation. From the above analysis and referring to Figure 2, a procedure of maintenance and service is proposed and complied as shown in Figure 3. From Figure 3, it is observed that optimal and robust operational quality can be obtained by adjusting CFs as long as there are no issues with PVs. However, if better performance improvement is required, further maintenance service can be undertaken through reviewing PVs, returning PVs to DPs, from DPs to FRs, or even from FRs to CAs for analyzing service value to satisfy customer's needs.

\section{Wire Bonder System}

\subsection{Mechatronics Structure}

The mechatronics functions of subsystems of a wire bonder indicate the functional responsibility by each component in a subsystem. The functionalities of the components of wire bonder equipment can be decomposed into different functional subsystems. For a wire bonder machine K\&S (Kulicke and Soffa) Max $\mu$ m Plus, the functional subsystems include Console System, Bond Head Assembly, Vision System, Material Handling System, XY-Table Assembly, Equipment Network, and Software. The functional subsystems are composed of different functional components of a mechatronics system. Console System includes sensing components, signal processing and controlling components, and power components. Bond Head Assembly includes driving components, motion executing components, and sensing components. Vision System includes sensing components. Mate- 
rial Handling System includes driving components, motion executing components, and sensing components. XY-Table Assembly includes driving components, motion executing components, and sensing components. Equipment Network and Software includes the network communication components, operating system components and also the application software components. The Equipment Network which is constructed by employing Semiconductor Equipment Communication Standard (SECS), General Equipment Model (GEM), and High Speed Message Series (HSMS) follows the Semiconductor Equipment Materials International (SEMI) standards. From the analysis, a three levels structure diagram is depicted as shown in Figure 4. The first level is functional subsystems. The second level is components of the corresponding subsystems. The third level is functional elements corresponding to each component. By utilizing the diagram of Figure 4, the mapping of a wire bonder from FRs to DPs

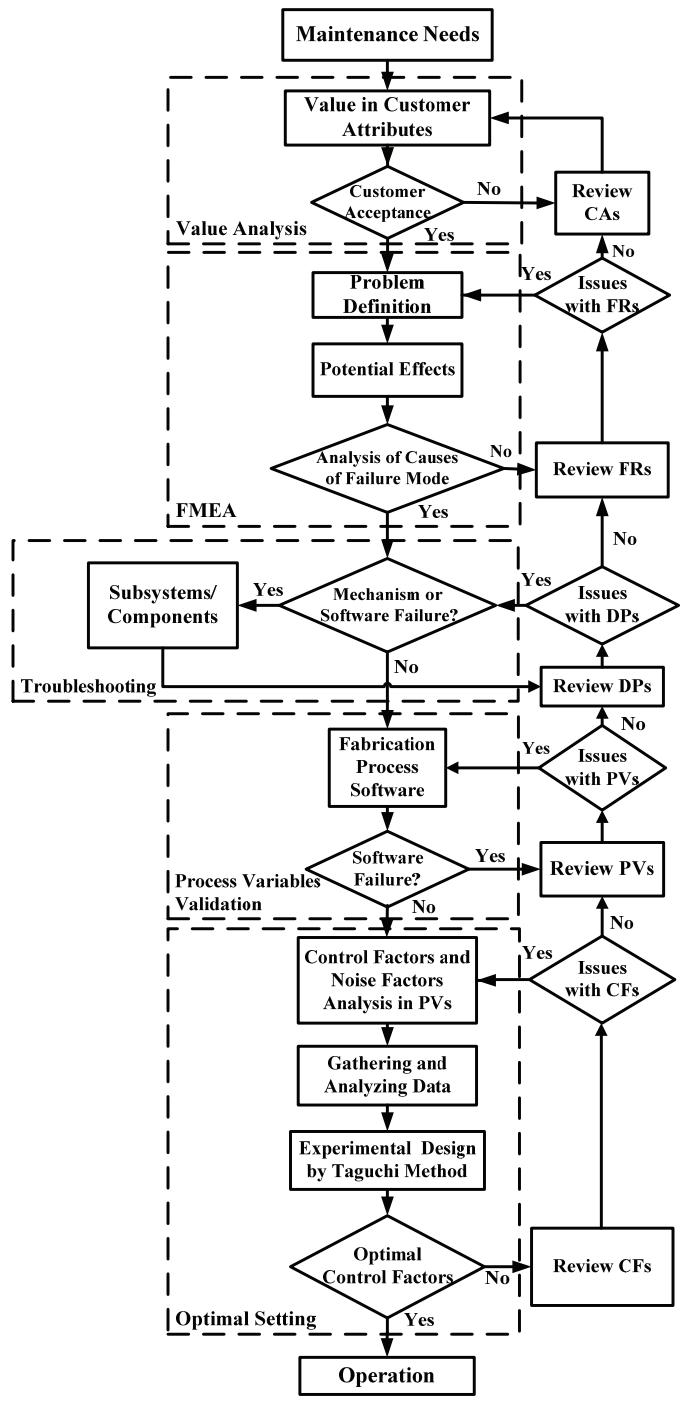

Figure 3. Procedure for maintenance and service can be analyzed and documented as machine knowledge in Figure 1.

\subsection{Knowledge Structure}

A wire bonder with five modules of subsystem is required to satisfy functional requirements. The mapping between FRs and DPs which satisfies a decoupled module design is given by (1) with weighting $A_{i j}$ as

$$
\left\{\begin{array}{l}
F R 1 \\
F R 2 \\
F R 3 \\
F R 4 \\
F R 5
\end{array}\right\}=\left[\begin{array}{ccccc}
A_{11} & 0 & 0 & 0 & 0 \\
A_{21} & A_{22} & 0 & 0 & 0 \\
A_{31} & A_{32} & A_{33} & 0 & 0 \\
A_{41} & A_{42} & 0 & A_{44} & 0 \\
A_{51} & A_{52} & 0 & 0 & A_{55}
\end{array}\right]\left\{\begin{array}{l}
D P 1 \\
D P 2 \\
D P 3 \\
D P 4 \\
D P 5
\end{array}\right\}
$$

The FRs in (1) are listed as

FR1: Provides command control and electronic control of equipment

FR2: Affords bonding motion for each recipe selected

FR3: Recognizes the image of materials by operator and machine

FR4: Provides material transportation capabilities

FR5: Maintains bonding table movement over the working area

The DPs for the subsystems in (1) are listed as

DP1: Signal, motion, and power control in Console System

DP2: Wire bonding is accomplished by mechanisms and controls of Bond Head Assembly

DP3: Targeting of devices is accomplished by Vision System

DP4: Move the materials for bonding demands as Material Handling System

DP5: XY-Table Assembly moves bonding mechanisms over the working space

\subsection{Knowledge Representation}

The knowledge representation constructed by axiomatic mapping can be derived from decomposition and zigzag mapping [9]. Only two levels in the representation of equipment knowledge are given in the following list. The lower levels of FRs and DPs will be given in the appendix.

1) Console System

FR1-1: All subsystems to be under control

DP1-1: Monitor and control system

FR1-2: Supply energy resources

DP1-2: Power system

FR1-3: Control the operation of machine

DP1-3: Human/machine interface

FR1-4: Software operations and data storage

DP1-4: Storage system

FR1-5: Air supply and control

DP1-5: Pneumatic system

FR1-6: Feed wire for automatic bonding

DP1-6: Wire feed system

2) Bond Head Assembly 


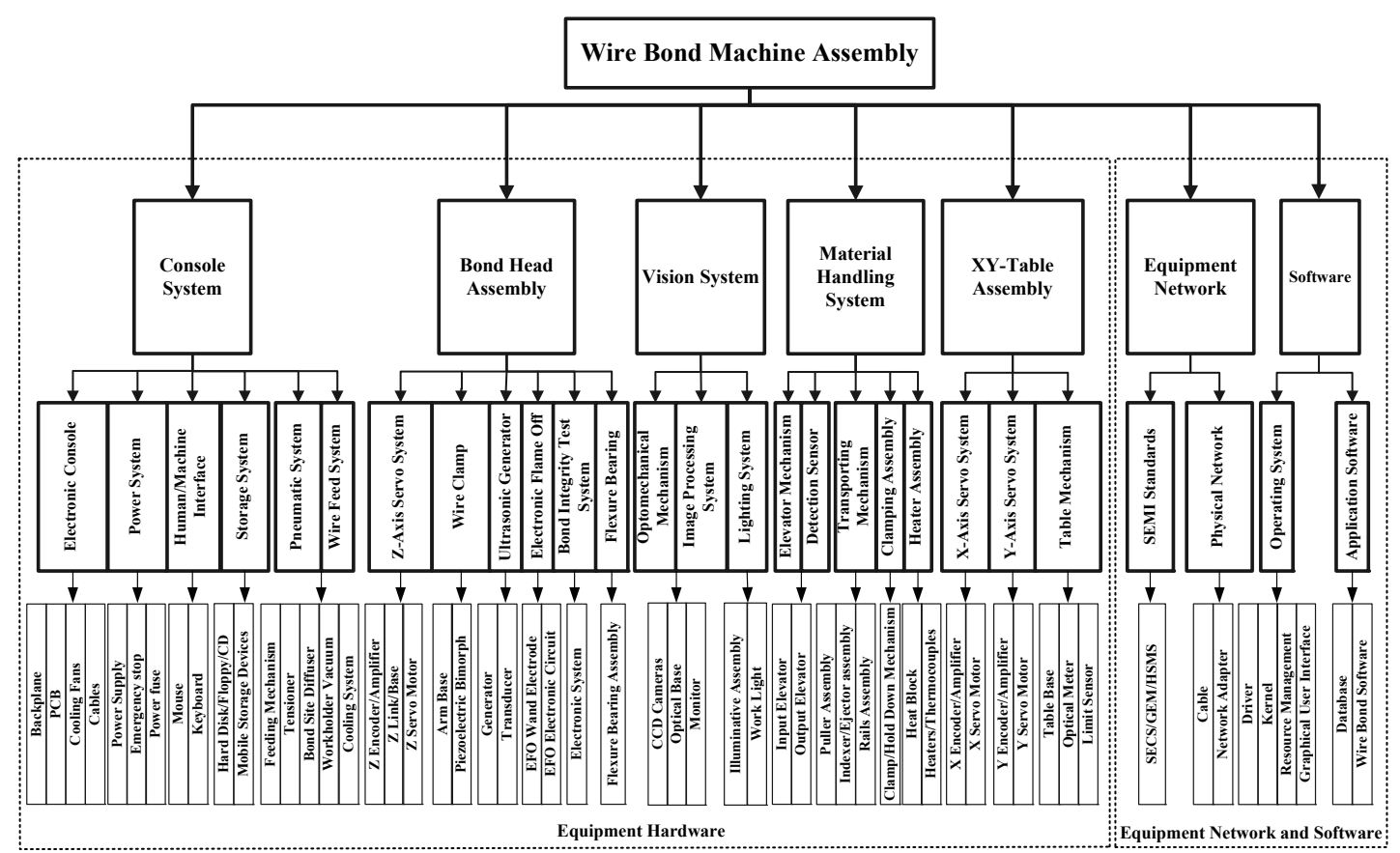

Figure 4. Structure diagram of a wire bonder

The following components are decomposed to be unique and independent and will be used as the basis for formulating DPs which are corresponding to FRs, respectively. The decomposition is undertaken for the Bond Head Assembly.

2.1 Electronic Flame Off (EFO)

FR2-1: Discharge for wire ball formation

DP2-1: EFO assembly

\subsection{Wire Clamp}

FR2-2: Feed and hold wire

DP2-2: Wire clamp assembly

2.3 Ultrasonic Generator (USG)

FR2-3: Generate and transmit ultrasonic energy

DP2-3: USG system

2.4 Z-axis Servo System

FR2-4: Bond head positioning and force control

DP2-4: Z-axis servo system

2.5 Bond Integrity Test System (BITS)

FR2-5: Detection of bonding outcome

DP2-5: Bond integrity test system

2.6 Flexure Bearing Assembly

FR2-6: Provide rotational movement

DP2-6: Flexure bearing

3) Vision System

The decomposition is undertaken for the Vision System with DP3 corresponding to FR3.

FR3-1: Clear image

FR3-2: Image acquisition with high speed

FR3-3: Focus range and field of view extension

DP3-1: Illumination and image acquisition system

DP3-2: Image processing system
DP3-3: Optomechanical extension mechanisms

4) Material Handling System

The decomposition is undertaken for the Material Handling System with DP4 corresponding to FR4.

FR4-1: Carry the materials in the bonding cycle

FR4-2: Fix the materials on the bond site

FR4-3: Provide the working temperature

FR4-4: Prevent materials damaged during transportation

FR4-5: Handle magazines during the bonding operation

DP4-1: Transporting mechanism

DP4-2: Clamping assembly

DP4-3: Heater assembly

DP4-4: Detection sensor

DP4-5: Elevator mechanism

5) XY-Table Assembly

The decomposition is undertaken for the XY-Table Assembly with DP5 corresponding to FR5.

FR5-1: I/O density of IC chip become higher

FR5-2: Chip dimension and the bond pad pitch become smaller

DP5-1: High speed and steady XY-servo system

DP5-2: XY-Table mechanism

\section{First Bond Failure and Maintenance}

By employing the design mappings combined with Mechatronics Engineering and engineer experience in representing the knowledge of a wire bonder, the failure modes regarding the first bond operation and the effects of various components on performance can be analyzed. 


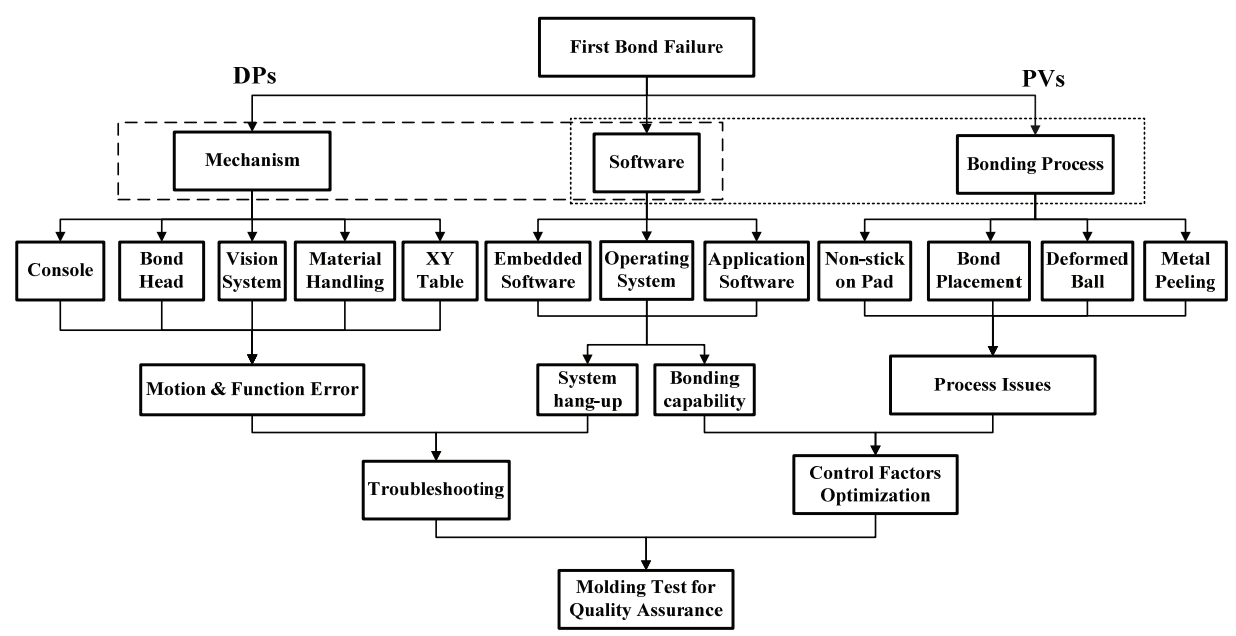

Figure 5. First bond failure analysis

The analysis of the first bond failure through DPs and PVs by the present approach is shown in Figure 5. By utilizing both engineering experience and documented equipment knowledge, the results of wire bonder failure modes corresponding to DPs are analyzed and obtained. In addition to these information and knowledge, the implementation results still rely on experience of measurements, tests, record of FRs, and customer feedback.

\subsection{Value Analysis}

Value is the first consideration by customers in needing maintenance as shown in Figure 3. In considering the CAs in needing maintenance service, a maintenance task [6] including task description, elapsed time, estimated cost for achieving specified functional performance will be proposed. With the proposed maintenance task analysis, the maintenance value can be justified and managed by customers. By assuming that the proposed service can satisfy CAs, the maintenance on the first bond failure will be undertaken by following Figure 3 through FMEA, Troubleshooting, Process Variables Validation, and Optimal Setting.

\subsection{FMEA}

In wire bonder industry, the performance of first bond operation can be qualified by employing the tests of ball size, ball shear, intermetallic compound, and etching. For the first bond operation, four common failure modes as Non-stick on Pad, Bond Placement, Deformed Ball, and Metal Peeling are identified as shown in Figure 5.

1) First Bond Failure Modes

1.1 Failure mode I : Non-Stick on Pad

Ball shear stress calculated with the apparent bonded area will provide a conservative underestimation of the quality of the gold-to-aluminum intermetallic bond line. This failure mode means a bond ball lift out of pad.

1.2 Failure mode $\Pi$ : Bond Placement
The accuracy of bond position must be controlled for the bond placement under the bond pad size.

1.3. Failure mode III: Deformed Ball

An ideal bond ball should have the best bond line quality with ball size proper for the bond pad.

1.4. Failure mode IV: Metal Peeling

Bond pad metal peeling is recognized as a serious problem of bonding ability and ball bond reliability in electronic packaging industry.

2) RPN Analysis

By analyzing the RPN value of the first bond failure, a decision making on the priority in maintenance can be justified and managed. The RPN for four failure modes is evaluated. The RPN is calculated as a mathematical product of the numerical ratings of severity, probability of occurrence, and detection likelihood given by

$$
A_{i-j, k}=s_{k} \times r_{i-j, k} \times m_{k} \times e_{i-j, k} \times n_{k} \times f_{i-j, k}
$$

In (2), the indices $i, j, k$ represent the sub-system, the component of the sub-system, and the failure mode, respectively. The $A_{i-j, k}$ is the RPN for each DPs of the failure mode $k$. The $s_{k}$ is a severity rating for failure mode $k$ and the $r_{i-j, k}$ is a severity rating for each DPs of the failure mode $k$. The $m_{k}$ is a probability rating for failure mode $k$ and the $e_{i-j, k}$ is a probability rating for each DPs of the failure mode $k$. The $n_{k}$ is a detection rating for failure mode $k$ and the $f_{i-j, k}$ is a detection rating for each DPs of the failure mode $k$. The RPN is calculated by assigning potential failure modes ranking from 1 to $10 \mathrm{with}$ respect to the severity of the failure mode effect, its probability of occurrence, and the likelihood of its being detected $[14,15]$. The assignment of ratings in the present study is according to the classification by SAE J1739 [15]. In applications, these ranking factors are highly dependent on the attributes of the system in operation. For providing a direct comparison of failure effects, the RPN value is normalized to give 


$$
B_{i-j, k}=A_{i-j, k} / \sum_{i, j} A_{i-j, k}
$$

The results of RPN ratio for the four kinds of failure modes of the first bond process are to be computed by utilizing (2) and (3) with data from Table 1.The results of RPN ratio with respect to DPs for the four failure modes are calculated and obtained as shown in Figure 6. From Figure 6, the optimal decision and associated actions can be inferred. In Figure 6, DPs with higher RPN

Table 1. Rating factors for evaluation of RPN in the first bond failure modes

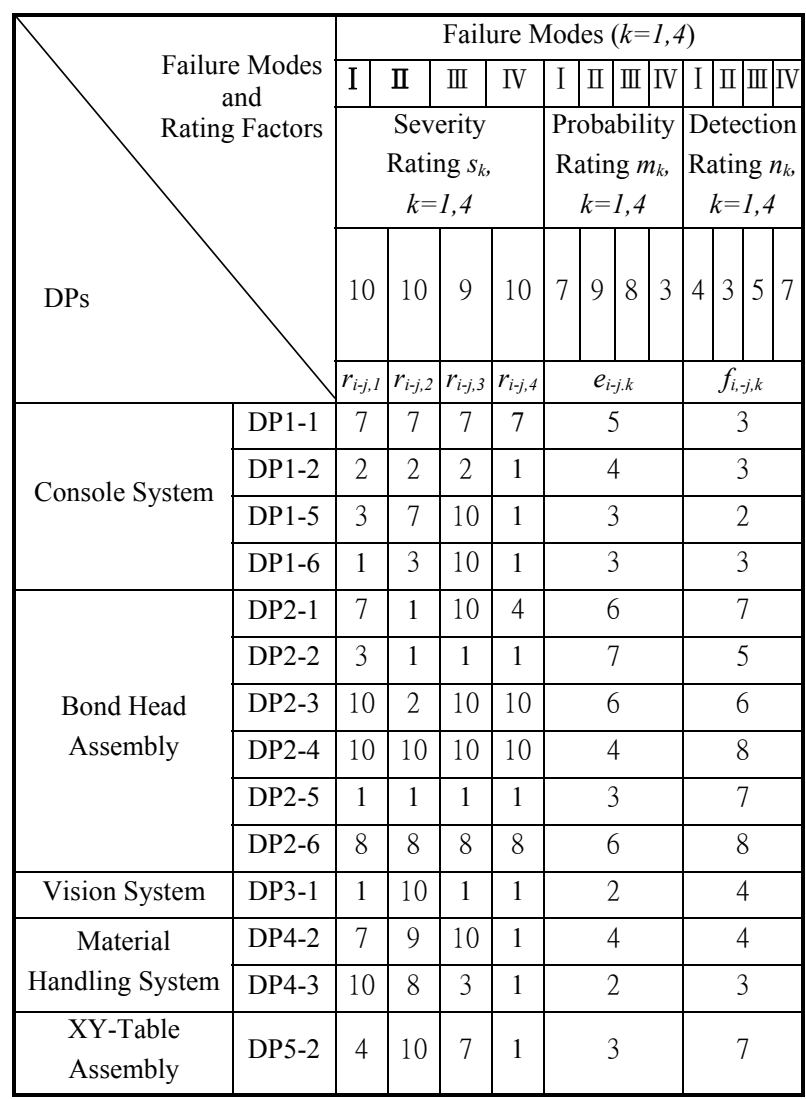

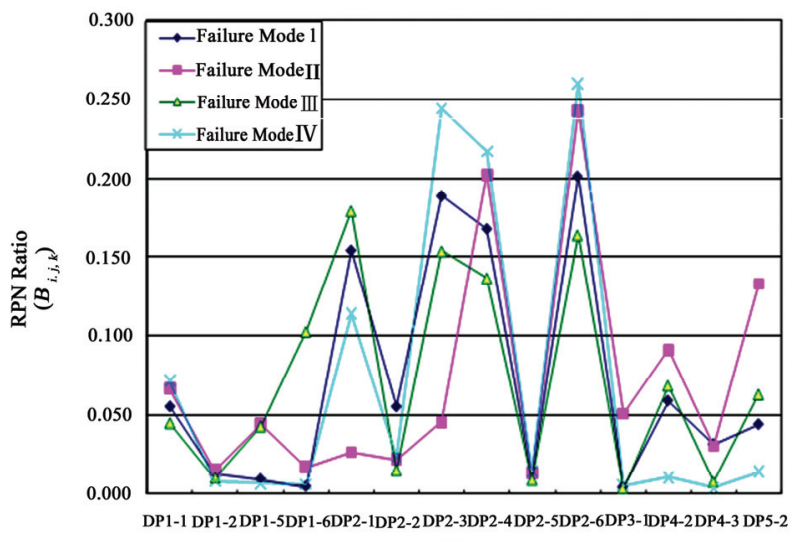

DPs

Figure 6. RPN ratio of maintenance for first bond process ratio for each failure mode are assumed to be more important and given higher maintenance priority than those having a lower RPN ratio. From Figure 6, it is observed that the highest priority for failure mode $I, I I$, and IV is DP2-6. The DP2-6 is a flexure bearing. For failure mode III, the highest priority is DP2-1. The DP2-1 is an EFO assembly. The priority distribution of RPN ratio for the components of wire bonder provides enough information for maintenance engineers to realize the effects of failure modes.

\subsection{Troubleshooting}

If there is no failure in the Software of Figure 5, the causes for the failure of DPs in the mechanism of the wire bonder are obtained by utilizing the knowledge representation and engineering experience in Figure 5 to give the following list.

1) Console System: failure related to mode I , II, and III

DP1-1: Monitor and control system

DP1-2: Power system

DP1-5: Pneumatic system

DP1-6: Wire feed system

2) Bond Head Assembly: failure related to mode I, II, III, and IV

DP2-1: EFO assembly

DP2-2: Wire clamp assembly

DP2-3: USG system

DP2-4: Z-axis servo system

DP2-5: Bond integrity test system

DP2-6: Flexure bearing

3) Vision System: failure related to mode $\Pi$

DP3-1: Illumination and image acquisition system

4) Material Handling System: failure related to mode I , II , and III

DP4-2: Clamping assembly

DP4-3: Heater assembly

5) XY-Table Assembly: failure related to mode $I I$ and III

DP5-2: XY-Table mechanism

From the analysis of DPs in the knowledge representation, causes analysis, and utilizing engineering experience for the first bond failure, the detail troubleshooting result of DPs is obtained as shown in Figure 7. The lower levels of DPs in Figure 7 are referred to Appendix. In Figure 7, the DPs are employed as DP1 for Console System, DP2 for Bond Head Assembly, DP3 for Vision System, DP4 for Material Handling System, and DP5 for XY-Table Assembly.

\subsection{Process Variables Validation}

From Figure 7, the related PVs will be analyzed through the mapping from DPs to PVs [9]. The PVs related to the bonding process in the maintenance and service of equipment operation can be analyzed, inferred, tested, and verified. For the first bond process, the environment 


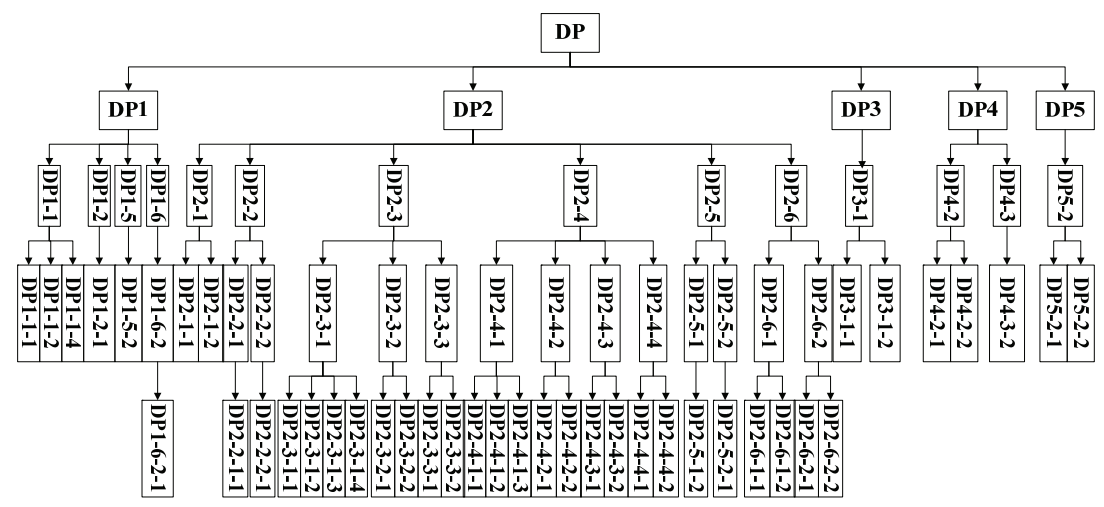

Figure 7. First bond failure troubleshooting

is met by the production standard. Bonding material and bonding temperature are fixed. A gold wire of $20 \mu \mathrm{m}$ is used. The temperature of bond-site area is $150^{\circ} \mathrm{C}$. Suitable capillary is chosen on $\mathrm{K} \& \mathrm{~S}$ Max $\mu \mathrm{m}$ Plus wire bonder. From the results obtained for the process study, the feedback of quality analysis responds the relationships between PVs and DPs. The PVs related to the process of the first bond failure are validated to give

PV1-1: Signal to electronic devices

PV1-2: Different rating of supply voltages

PV1-3: Different input commands

PV1-4: Different program storage selections

PV1-5: System flow rate adjustments

PV1-6: Wire feed flow rate adjustments

PV2-1: Adjustment of EFO firing time and control current

PV2-2: Adjust wire pullout

PV2-3: Setting ultrasonic power utilization

PV2-3-1: Setting USG applied amplitude and frequency

PV2-3-2: Capillary tube and transducer installation adjustment

PV2-3-3: Setting USG in bonding process

PV2-3-3-1: Setting pre-bleed USG

PV2-3-3-2: Setting bonding USG

PV2-4: Variables in Z-axis motion on bonding time and force

PV2-4-1: Bonding force

PV2-4-2: Z-axis position

PV2-4-3: Bonding time

PV2-4-4: Capillary searching speed

PV2-5: Signal of bonding processes

PV3-1: Adjust optical limit and select image setting

PV3-2: Set parameters of image processing

PV3-3: Select different cameras and magnifications

PV4-1: Material transportation variables

PV4-2: Clamping compensation adjustments

PV4-3: Variables of bonding temperature

PV4-4: Set up sensor sensitivity

PV4-5: Adjust elevator parameters

PV5-1: Adjust XY-axes motion and speed
PV5-2: Adjust XY-motion accuracy

\subsection{Optimal Setting}

Minimization of the variation of the bonding process is to achieve high yield in production. The specifications of bond ball can be set according to customer's requirements. Optimization of CFs for assuring bond ball quality is undertaken by employing Taguchi method $[12,13]$. In the Taguchi's experimental tests, the quality target is called signal and the variation in the target is called noise.

1) Quality Characteristics

For the first bond process, the quality requirements of ball size and ball shear are specified. The quality target of ball size, as tri-axial appearances, is given as Nominal-the-Best. The quality target of ball shear force is given as The-Larger-the-Better. The shear force is obtained by utilizing shear strength, as shear force per unit area, multiplied by the estimated area. The quality specifications for the first bond in the experiment are given as

1.1. Quality Targets:

a. Ball size: Nominal-the-Best

Ball X \& Ball Y (Plane of bond pad) $-40 \mu \mathrm{m}+/-2 \mu \mathrm{m}$

Ball Z (Plane orthogonal to bond pad) $-10 \mu \mathrm{m}$ $+/-2 \mu \mathrm{m}$

b. Ball shear: The-Larger-the-Better

Ball shear force-Above 12 grams

1.2. Portability of Quality:

For the requirement of portability of quality, experimental tests need large sample size, different machine bonding, and high productive yield.

2) Control Factors Analysis

The function of a wire bonder is assumed correct. The $\mathrm{CFs}$ influencing the quality of bond ball forming are selected. For the first bond, the experimental CFs use Constant Velocity in Capillary Searching Speed (PV2-4-4), Bonding USG (PV2-3-3-2), Bonding Time (PV2-4-3), Bonding Force (PV2-4-1), and Pre-Bleed USG (PV23-3-1) to verify failure modes I and III.

3) Experimental Tests and Confirmation 
The experimental tests are undertaken in laboratory by the combination of five CFs and different levels as shown in Table 2. For portability tests, three devices are separately bonded on two machines. For one factor with two levels and four factors with three levels in combination, the experimental results are based on L18 orthogonal arrays and listed in Table 3.

By following the procedure of Taguchi method [13], the tables and graphs of factor response are obtained and employed to show $S / N$ ratio for different combinations of parameters. The $S / N$ ratio for the Nominal-the-Best is calculated by (4) with the mean and standard deviation of data $y_{i}$, as

$$
S / N=-10 \log \left(\frac{\sigma^{2}}{\bar{y}^{2}}\right)
$$

The $S / N$ ratio for The-Larger-the-Better is calculated by (5) as

$$
S / N=-10 \log \frac{\sum_{i=1}^{n} \frac{1}{y_{i}^{2}}}{n}
$$

From Figure 8, it is observed that the variations of bonding time for the quality targets are small. Thus, the bonding time is considered as an adjustable factor and selected as $\mathrm{C} 1$. From the $S / N$ ratio obtained in Figure 8, the final optimal combinations are inferred by four sets of parameters in sequence as [A1 B2 C1 D2 E2], [A1 B3 C1 D2 E2], [A1 B2 C1 D2 E3], [A1 B3 C1 D2 E3]. Al-

Table 2. Control factors and levels for first bond process.

\begin{tabular}{|c|r|c|c|c|}
\hline Factor & Variables Description & Level 1 & Level 2 & Level 3 \\
\hline 1 & $\begin{array}{r}\text { A: Capillary Searching } \\
\text { Speed (mil/ms) }\end{array}$ & 0.1 & 0.14 & - \\
\hline 2 & B: Bonding USG (mA) & 80 & 90 & 100 \\
\hline 3 & C: Bond Time (ms) & 8 & 14 & 20 \\
\hline 4 & D: Bond Force (grams) & 15 & 25 & 35 \\
\hline 5 & E: Pre-Bleed USG (\%) & 25 & 45 & 65 \\
\hline
\end{tabular}

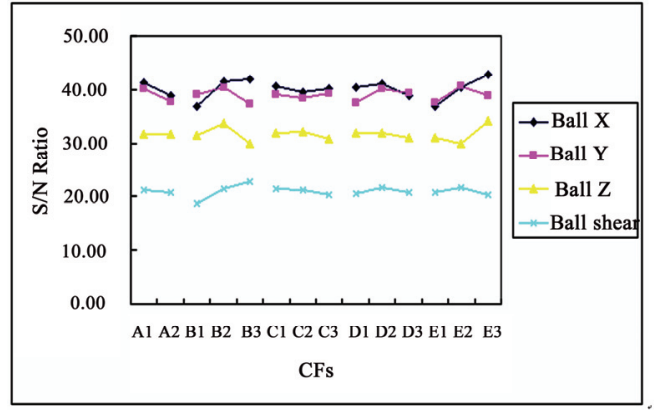

Figure 8. Overall factors response

Table 4. Comparisons of $\mathrm{S} / \mathrm{N}$ ratio for four sets of optimal combination

\begin{tabular}{|c|c|c|c|c|c|}
\hline \hline Optimal Set & S/N Ratio & Ball X & Ball Y & Ball Z & $\begin{array}{c}\text { Ball } \\
\text { Shear }\end{array}$ \\
\hline \multirow{2}{*}{ Set 1} & Prediction & 44.53 & 44.47 & 32.41 & 23.83 \\
\cline { 2 - 6 } & Experiment & 42.74 & 45.12 & 38.33 & 30.48 \\
\hline \multirow{2}{*}{ Set 2} & Prediction & 45.11 & 41.29 & 28.65 & 25.01 \\
\cline { 2 - 6 } & Experiment & 41.4 & 43.15 & 32.33 & 29.84 \\
\hline \multirow{2}{*}{ Set 3} & Prediction & 46.92 & 42.74 & 36.67 & 22.42 \\
\cline { 2 - 6 } & Experiment & 44.25 & 42.03 & 32.02 & 28.31 \\
\hline \multirow{2}{*}{ Set 4} & Prediction & 47.5 & 39.56 & 32.91 & 23.6 \\
\cline { 2 - 6 } & Experiment & 46.77 & 43.47 & 31.53 & 30.1 \\
\hline
\end{tabular}

though there are four sets of optimal parameters in solution, a set of optimal parameters will be selected by confirmation experiments. The confirmation test is undertaken by the fabrication of ten devices under four sets of optimal combinations respectively. Four sets of confirmation for the experimental and predicted $S / N$ are obtained as given in Table 4. In Table 4, the experimental result is calculated from the mean $S / N$ ratio of testing results and the prediction of $S / N$ ratio is obtained by utilizing a linear additive predictive model. The confirmation test reveals that the quality targets are satisfied by all the four sets of optimal combination. By comparing the

\begin{tabular}{|c|c|c|c|c|c|c|c|c|c|c|c|c|c|c|c|c|c|c|c|c|c|c|c|c|c|c|c|c|c|}
\hline \multicolumn{30}{|c|}{ Experimental Data } \\
\hline \multirow{3}{*}{ No. } & \multicolumn{5}{|c|}{ Control Factors } & \multicolumn{12}{|c|}{ M1 (Machine \#1) } & \multicolumn{12}{|c|}{ M2 (Machine \#2) } \\
\hline & \multirow{2}{*}{\begin{tabular}{c|}
$\mathrm{CN}$ \\
$\mathrm{A}$
\end{tabular}} & \multirow{2}{*}{\begin{tabular}{|c|} 
USG \\
$\mathrm{B}$ \\
\end{tabular}} & \multirow{2}{*}{\begin{tabular}{|c|} 
Time \\
$\mathrm{C}$ \\
\end{tabular}} & \multirow{2}{*}{\begin{tabular}{|c|} 
Fore \\
D \\
\end{tabular}} & \multirow{2}{*}{\begin{tabular}{c|} 
Pre- \\
Bleed \\
E
\end{tabular}} & \multicolumn{4}{|c|}{ y1(Device \#1) } & \multicolumn{4}{|c|}{ y2(Device \#2) } & \multicolumn{4}{|c|}{ y3(Device \#3) } & \multicolumn{4}{|c|}{ y1(Device \#1) } & \multicolumn{4}{|c|}{ y2(Device \#2) } & \multicolumn{4}{|c|}{ y3(Device \#3) } \\
\hline & & & & & & Ball & Ball y & Ball $z$ & \begin{tabular}{|l|l|} 
Ball- \\
Shless
\end{tabular} & Ball $x$ & Ball y & Ball $\mathrm{z}$ & \begin{tabular}{|c|c|} 
Ball- \\
Sheer \\
\end{tabular} & Ball $\mathrm{x}$ & Ball y & Ball z & \begin{tabular}{|l|} 
Ball- \\
Shear \\
\end{tabular} & & Bally & Ball $z$ & Shearl & & Ball y & Ball $z$ & \begin{tabular}{|l|l|} 
Ball- \\
Sheesu \\
\end{tabular} & Ball $x$ & Ball y & & $=\left.\right|_{\text {Shear }} ^{\text {Ball- }}$ \\
\hline 1 & 1 & 1 & 1 & 1 & \begin{tabular}{|l|l}
1 \\
\end{tabular} & 38 & 37.8 & 14.5 & 8.59 & 38.8 & 37.3 & 14.2 & 8.45 & 38.4 & 36.8 & 14.4 & 7.25 & 37.3 & 37.4 & 15.2 & 9.85 & \begin{tabular}{|l|l}
38.6 & 3 \\
\end{tabular} & 38.2 & 14.5 & 9.23 & 37.9 & 38 & 14.8 & 9.11 \\
\hline 2 & 1 & 1 & 2 & 2 & 2 & 39.8 & 39.3 & 12.3 & 10.78 & 40.5 & 39.2 & 12.5 & 11.46 & 39.5 & 38.9 & 12.2 & 10.11 & 39.7 & 39.4 & 12.4 & 9.37 & \begin{tabular}{|l|l}
40.3 \\
\end{tabular} & \begin{tabular}{|l|l}
39.1 \\
\end{tabular} & 11.6 & 10.37 & 39.4 & 39.3 & 11.8 & 11.12 \\
\hline 3 & 1 & 1 & 3 & 3 & 3 & $\begin{array}{l}43.2 \\
\end{array}$ & 42.8 & 8.4 & 7.61 & 43.5 & \begin{tabular}{|l|}
42.7 \\
\end{tabular} & \begin{tabular}{|l|}
8.1 \\
\end{tabular} & 6.17 & \begin{tabular}{|l|l|}
43.7 \\
\end{tabular} & \begin{tabular}{|l|}
43 \\
\end{tabular} & \begin{tabular}{|l|}
8.6 \\
\end{tabular} & 8.64 & 44.5 & \begin{tabular}{|l|l|}
42.2 \\
\end{tabular} & 79 & 7.76 & \begin{tabular}{|l|l}
42.5 & 4 \\
\end{tabular} & \begin{tabular}{l|l|}
42.2 \\
\end{tabular} & \begin{tabular}{|l|}
8.2 \\
\end{tabular} & 8.64 & 43.6 & 42.5 & \begin{tabular}{|l|l|}
7.8 \\
\end{tabular} & 8.53 \\
\hline 4 & 1 & 2 & 1 & 2 & 3 & 40.1 & 40.1 & 12.6 & 12.84 & 40.2 & 39.4 & 12.5 & 12.60 & 40.4 & 40.3 & 12.6 & 12.63 & 40.1 & 39.4 & 12.4 & 12.54 & \begin{tabular}{|l|l|l|}
40.2 & 3 \\
\end{tabular} & 39.8 & 12.3 & 13.19 & 40.3 & 40.2 & 12.6 & 11.58 \\
\hline 5 & 1 & 2 & 2 & 3 & 1 & 41.6 & 42.3 & 10.6 & 13.24 & 41.3 & 42.6 & 10.8 & 13.32 & 41.7 & 42.8 & 10.6 & 14.77 & \begin{tabular}{|l|l|}
41.8 \\
\end{tabular} & 41.7 & 10.3 & 12.94 & \begin{tabular}{|l|l|l|l|}
41.9 & 4 \\
\end{tabular} & 42.3 & 10.2 & 12.31 & 42.8 & 42.5 & 10.3 & 13.22 \\
\hline 6 & 1 & 2 & 3 & 1 & 2 & 38.5 & 38.2 & 12.5 & 11.21 & 38.4 & 38.9 & 11.7 & 11.05 & 38.7 & 38.3 & 12.6 & 11.20 & \begin{tabular}{|l|}
38.4 \\
\end{tabular} & 38.5 & 12.7 & 11.21 & \begin{tabular}{|l|l|l|}
38.5 & 3 \\
\end{tabular} & 38.1 & $\mid 12.2$ & 11.23 & 38.1 & 38.5 & \begin{tabular}{|l|}
12.3 \\
\end{tabular} & 11.05 \\
\hline 7 & 1 & 3 & 1 & 3 & 2 & \begin{tabular}{|l|}
43.1 \\
\end{tabular} & 42 & \begin{tabular}{|l|}
8.4 \\
\end{tabular} & 15.85 & 43 & \begin{tabular}{|l|}
42 \\
\end{tabular} & 79 & 15.71 & \begin{tabular}{|l|l|}
43.1 \\
\end{tabular} & \begin{tabular}{|l|}
41.7 \\
\end{tabular} & \begin{tabular}{|l|}
8.2 \\
\end{tabular} & 15.95 & 43.5 & 42.5 & 8.5 & 15.97 & \begin{tabular}{|l|l}
43.4 & 4 \\
\end{tabular} & \begin{tabular}{|l|l|}
42.7 \\
\end{tabular} & \begin{tabular}{|l|}
8.2 \\
\end{tabular} & 15.99 & 43.6 & \begin{tabular}{|l|}
42.4 \\
\end{tabular} & 8.6 & 15.66 \\
\hline 8 & 1 & 3 & 2 & 1 & 3 & 39.8 & 39.9 & 12.5 & 12.01 & 40.7 & 39.8 & 12.5 & 12.31 & 40 & 39.4 & 12 & 12.30 & 40 & 39.3 & 11.8 & 11.16 & 39.8 & \begin{tabular}{|c|c|}
39 \\
\end{tabular} & 12.5 & 11.36 & 39.9 & 41.1 & 12.2 & 11.54 \\
\hline 9 & 1 & 3 & 3 & 2 & 1 & 41.4 & 40.5 & 10.5 & 14.60 & 41.2 & 39.8 & 9.6 & 14.7 & 42.3 & 40.7 & 10.4 & 14.74 & 41 & 40.7 & 10.8 & 14.52 & 42.2 & 42 & $\mid 10.7$ & 14.94 & 41.6 & 40.8 & 10 & 14.76 \\
\hline 10 & 2 & 1 & 1 & 1 & 1 & 36.5 & 37.6 & 15.1 & 8.98 & \begin{tabular}{|l|}
36.2 \\
\end{tabular} & 36.3 & \begin{tabular}{|l|}
15.6 \\
\end{tabular} & \begin{tabular}{|l|}
8.86 \\
\end{tabular} & \begin{tabular}{|l|}
38.7 \\
\end{tabular} & \begin{tabular}{|l|}
38.3 \\
\end{tabular} & \begin{tabular}{|l|}
14.8 \\
\end{tabular} & 8.06 & \begin{tabular}{|c|}
37.1 \\
\end{tabular} & 37.2 & \begin{tabular}{|l|}
14.4 \\
\end{tabular} & 8.06 & \begin{tabular}{|l|l}
36.4 & 3 \\
\end{tabular} & 37.3 & \begin{tabular}{|l|}
15.3 \\
\end{tabular} & \begin{tabular}{|l|}
7.67 \\
\end{tabular} & 36.3 & \begin{tabular}{|l|}
37.1 \\
\end{tabular} & 14.6 & \begin{tabular}{|l|} 
\\
\end{tabular} \\
\hline 11 & 2 & 1 & 2 & 2 & 2 & \begin{tabular}{|l|l|}
39.1 \\
\end{tabular} & 39.7 & \begin{tabular}{|l|}
11.3 \\
\end{tabular} & 10.55 & 38.8 & 38.9 & \begin{tabular}{|l|}
10.7 \\
\end{tabular} & 9.44 & \begin{tabular}{|l|l|}
39.8 \\
\end{tabular} & 39.6 & \begin{tabular}{|l|}
11 \\
\end{tabular} & 10.09 & 38.8 & 39.1 & 11.5 & 11.29 & \begin{tabular}{|l|l|}
39.4 & 3 \\
\end{tabular} & 39.9 & \begin{tabular}{|l|}
11.4 \\
\end{tabular} & \begin{tabular}{|l|l|}
11.14 \\
\end{tabular} & 40.3 & \begin{tabular}{|l|}
40.7 \\
\end{tabular} & 10.7 & 11.03 \\
\hline 12 & 2 & 1 & 3 & 3 & 3 & \begin{tabular}{|l|}
42.5 \\
\end{tabular} & 42.9 & \begin{tabular}{|l|}
9.7 \\
\end{tabular} & 6.80 & \begin{tabular}{|l|l|} 
\\
\end{tabular} & \begin{tabular}{|l|}
43.7 \\
\end{tabular} & \begin{tabular}{|l|}
9.8 \\
\end{tabular} & \begin{tabular}{|l|}
6.26 \\
\end{tabular} & 43.2 & \begin{tabular}{|l|}
43.1 \\
\end{tabular} & 9.6 & 6.57 & 43 & \begin{tabular}{|l|} 
\\
\end{tabular} & 9.5 & 6.69 & \begin{tabular}{|l|l}
42.3 & 4 \\
\end{tabular} & 42.4 & \begin{tabular}{|l|}
9.4 \\
\end{tabular} & 6.40 & 42.5 & 42 & 9.7 & 6.95 \\
\hline 13 & 2 & 2 & 1 & 2 & 3 & 39.6 & 38.9 & 12,4 & 13.36 & 40.1 & 39 & 11.9 & 13.01 & \begin{tabular}{|l|}
40.2 \\
\end{tabular} & 39.3 & 12.6 & 13.22 & 39.3 & 39.5 & \begin{tabular}{l|l}
12 \\
\end{tabular} & 12.70 & 39.5 & 38.8 & 12.2 & 13.07 & 39.5 & \begin{tabular}{|l|}
39.3 \\
\end{tabular} & 12.1 & 13.90 \\
\hline 14 & 2 & 2 & 2 & 3 & 1 & 42.2 & 41.9 & \begin{tabular}{|l|}
9.4 \\
\end{tabular} & 10.07 & 43 & 41.5 & \begin{tabular}{|l|}
9.2 \\
\end{tabular} & 10.44 & \begin{tabular}{|l|}
42.8 \\
\end{tabular} & 42.9 & 9.6 & 10.73 & 40.8 & 41.2 & 9.2 & \begin{tabular}{|l|}
9.48 \\
\end{tabular} & \begin{tabular}{|l|l}
42 & 4 \\
\end{tabular} & \begin{tabular}{|l|}
42.5 \\
\end{tabular} & \begin{tabular}{|l|}
9.6 \\
\end{tabular} & 9.97 & 42.2 & \begin{tabular}{|l|}
42.1 \\
\end{tabular} & 9.7 & 10.12 \\
\hline 15 & 2 & 2 & 3 & 1 & 2 & 36.9 & 37.8 & \begin{tabular}{|l|l}
13.1 \\
\end{tabular} & 11.40 & \begin{tabular}{|l|}
77.8 \\
\end{tabular} & \begin{tabular}{|l|}
37.6 \\
\end{tabular} & 12.8 & 11.88 & 38.3 & 38.1 & 12.9 & 11.39 & 37.7 & \begin{tabular}{|l|}
37.6 \\
\end{tabular} & 13.2 & 10.93 & \begin{tabular}{|l|}
77.6 \\
\end{tabular} & 37 & \begin{tabular}{|l|}
13.4 \\
\end{tabular} & 11.95 & \begin{tabular}{|l|}
37.8 \\
\end{tabular} & 37.3 & \begin{tabular}{ll|} 
& 13.7 \\
\end{tabular} & 11.07 \\
\hline 16 & 2 & 3 & 1 & 3 & 2 & 42.8 & 43.1 & \begin{tabular}{|l|}
8.8 \\
\end{tabular} & \begin{tabular}{|l|}
15.14 \\
\end{tabular} & \begin{tabular}{|l|} 
\\
\end{tabular} & \begin{tabular}{|l|l|} 
\\
\end{tabular} & \begin{tabular}{|l|l|}
8.4 \\
\end{tabular} & 14.35 & 43.1 & \begin{tabular}{|l|} 
\\
\end{tabular} & 8.6 & 15.55 & 42.2 & 42.8 & 8.6 & 15.95 & \begin{tabular}{|l|l}
41.9 & 4 \\
\end{tabular} & 42.3 & 9.6 & 14.73 & \begin{tabular}{|l|l|}
41.8 \\
\end{tabular} & 43.9 & 8.3 & 15.00 \\
\hline 17 & 2 & 3 & 2 & 1 & 3 & 40.2 & 38.7 & 12 & 12.72 & 39.9 & 38.5 & 11.5 & 12.96 & 39.8 & 38.1 & 12.1 & 13.49 & 39.8 & \begin{tabular}{|l|}
39.7 \\
\end{tabular} & 11.8 & 12.79 & \begin{tabular}{|l|l}
40.1 & 3 \\
\end{tabular} & 38.9 & \begin{tabular}{|l|l|}
11.6 \\
\end{tabular} & 11.46 & 39.9 & 38.1 & 11.9 & 12.56 \\
\hline 18 & 2 & 3 & 3 & 2 & 1 & \begin{tabular}{|l|}
41.8 \\
\end{tabular} & 40.4 & 11.4 & 12.35 & 42.2 & 40.5 & 11.5 & 12.05 & \begin{tabular}{|l|l|}
41.8 \\
\end{tabular} & \begin{tabular}{|l|}
40.2 \\
\end{tabular} & 11.6 & 12.95 & \begin{tabular}{|l|}
41.7 \\
\end{tabular} & 39.6 & 11.3 & \begin{tabular}{|l|}
11.93 \\
\end{tabular} & \begin{tabular}{|l|l|}
41.5 & 3 \\
\end{tabular} & \begin{tabular}{|l|l|}
39.7 \\
\end{tabular} & \begin{tabular}{|l|l|}
11.4 \\
\end{tabular} & 11.83 & \begin{tabular}{|l|}
41.2 \\
\end{tabular} & 40.6 & 10.6 & 12.18 \\
\hline
\end{tabular}

Table 3. Experimental orthogonal arrays and experimental data (K \& S Max $\mu \mathrm{m}$ Plus Wire Bonder) 
closeness of $S / N$ ratio of each set of prediction and experimental result in Table 4 , it is realized that the interactions of CFs are insensitive for Ball X and Ball Y, but the interactions for Ball $\mathrm{Z}$ and Ball Shear are sensitive.

With the interactions in laboratory tests, the optimal result obtained cannot be directly applied for customer service. In order to achieve optimal bonding performance and robustness in field operation, further experimental designs and tests are required [13]. For the present results obtained in laboratory, the results of ball size are acceptable by the quality target. The experimental $S / N$ ratio of Ball Shear for Set 1 is higher than the other three sets of experimental results. With the higher $S / N$ ratio in Set 1 , the wire bonding process will be more robust. From the experimental confirmation tests, the final optimal parameters for K\&S Max $\mu \mathrm{m}$ Plus are [A1 B2 C1 D2 E2], i.e. Capillary Searching Speed: $0.1 \mathrm{mil} / \mathrm{ms}$, Bonding USG: $90 \mathrm{mAmp}$, Bonding Time: $8 \mathrm{~ms}$, Bonding Force: 25 grams, and Pre-bleed USG: $45 \%$.

\section{Conclusions}

A systematic framework which consists of a system of equipment failure analysis, methods, process, and activities, and a procedure is proposed for the maintenance and service of semiconductor equipment. The maintenance procedure in sequence starting from Maintenance Needs, Value Analysis, FMEA, Troubleshooting, Manufacturing Process Validation, and Optimal Setting to Operation is developed. By employing axiomatic design mapping integrated with Value Engineering, Quality Function Deployment, Mechatronics Engineering, Techniques from R\&D and Supplier, and Taguchi Method, the framework is highly effective in translating Maintenance Needs into Design Parameters for troubleshooting, and into Control Factors for process optimization. In the troubleshooting and process optimization of a wire bonder, the framework is applied to assure robust production quality in the first bond process by K\&S Max $\mu \mathrm{m}$ Plus. The systematic framework provides an effective and efficient scheme to provide maintenance and service for the acceptance or worth by customers.

\section{Appendix}

The lower levels of FRs and DPs are listed as follows.

1) Console System

FR1-1-1: Mechanism motion control

FR1-1-2: Signal transmission

FR1-1-3: Image processing

FR1-1-4: Communication relationship

DP1-1-1: Electronic mechanism control

DP1-1-2: Sensor interfaces

DP1-1-3: Image processing system

DP1-1-4: Circuit connection and cabling

FR1-2-1: Provide power for all components

FR1-2-2: Circuit and mechanism protection
DP1-2-1: Power supply assembly

DP1-2-2: Electronic fuse and emergency stop

FR1-3-1: Motion movement, including function and message selections in the GUI

FR1-3-2: Command typing and entry of data

DP1-3-1: Mouse device

DP1-3-2: Keyboard

FR1-4-1: Bonder software and applications

FR1-4-2: Portable bond programs and applications

DP1-4-1: Hard disk

DP1-4-2: Mobile storage device (Floppy, USB storage device, CD ROM, etc.)

FR1-5-1: Mechanism protection

FR1-5-2: Fabrication process demand

FR1-5-3: Auxiliary machinery

DP1-5-1: Cooling air

DP1-5-2: Vacuum system and compressed air

DP1-5-3: Air pressure with mechanisms

FR1-6-1: Precise wire feed for bonding continuously

FR1-6-1-1: Control wire feed motion and timing

FR1-6-1-2: Feed wire automatically

FR1-6-2: Optimal bonding quality

FR1-6-2-1: Maintain the bond ball quality

FR1-6-2-2: Looping performance

DP1-6-1: Feeding mechanism

DP1-6-1-1: Motorized spool assembly with sensor detection

DP1-6-1-2: Air guide and wire detect sensor

DP1-6-2: Optimal wire feed path in pneumatic system

DP1-6-2-1: Tension the wire and hold the ball in the center of capillary

DP1-6-2-2: Optimizing air guide to reduce wire whipping

2) Bond Head Assembly

FR2-1-1: Required wire bonding quality by EFO

FR2-1-1-1: Fine Pitch bonding

FR2-1-1-2: High yield and bond ability

DP2-1-1: Three-phase controller of EFO.

DP2-1-1-1: Control of discharge energy of the EFO

DP2-1-1-2: Control the wear and lifetime of electrode

FR2-2-1: Proven reliability in operation

FR2-2-1-1: Eliminate motion wear

FR2-2-1-2: High operational times

FR2-2-2: Clamp performance

FR2-2-2-1: Active damping of clamping mechanical resonance

FR2-2-2-2: Shorter opening and closing time

DP2-2-1: Wire contact in handling and mounting

DP2-2-1-1: Compliant motion mechanism

DP2-2-1-2: Fixed arm and bimorph arm

DP2-2-2: Wire clamp driver with voltage profiling for open-loop control

DP2-2-2-1: Bimorph clamp actuator

DP2-2-2-2: Piezoelectric actuation

FR2-3-1: Efficient and effective ultrasonic energy 
FR2-3-1-1: Ball roundness

FR2-3-1-2: Fine pitch wire bonding capabilities

FR2-3-1-3: High speed wire bonding

FR2-3-1-4: Minimize the loss of ultrasonic energy

FR2-3-2: Good placement accuracy

FR2-3-2-1: Higher bonding accuracy

FR2-3-2-2: Good stability and reproducibility of capillary tip motion

FR2-3-3: Good bonding capability

FR2-3-3-1: USG applied before capillary contact substrate

FR2-3-3-2: USG applied during contact and bonding operation

DP2-3-1: Generation, transmission and focusing mechanism of ultrasonic energy

DP2-3-1-1: Accurate ultrasonic amplitude and frequency

DP2-3-1-2: High frequency vibration of PZT

DP2-3-1-3: Increase system stiffness by using titanium

DP2-3-1-4: Holder and horn mechanism

DP2-3-2: Vibration of transducer tip

DP2-3-2-1: Transducer material and structure

DP2-3-2-2: Capillary clamping system of the transducer

DP2-3-3: Ultrasonic energy by USG in bonding process

DP2-3-3-1: Capillary tube and ultrasonic profile

DP2-3-3-2: Bonding ultrasonic control circuitry

FR2-4-1: Precise motion and force control on bond head

FR2-4-1-1: Capable of high acceleration

FR2-4-1-2: High motion repeatability

FR2-4-1-3: Bonding accuracy

FR2-4-2: Monitor Z-axis position for force control

FR2-4-2-1: Monitor bonding tool position precisely

FR2-4-2-2: Real-time signals response

FR2-4-3: Speed control of bond head

FR2-4-3-1: Higher acceleration

FR2-4-3-2: High performance of control system

FR2-4-4: Capillary and wire motion control

FR2-4-4-1: Detecting and switching the approaching to substrate

FR2-4-4-2: Searching speed requirement

DP2-4-1: Voice coil actuating control system

DP2-4-1-1: Minimize the mass of the motor and balance the centre of gravity of the rotational inertia.

DP2-4-1-2: Electromagnetic and mechanical subsystems

DP2-4-1-3: Accurate servo mechanism

DP2-4-2: Encoder assembly

DP2-4-2-1: High resolution optical grid

DP2-4-2-2: Electronic encoder system

DP2-4-3: Speed control system

DP2-4-3-1: Low inertia mechanism

DP2-4-3-2: High bandwidth in control loop
DP2-4-4: Capillary and wire motion control system

DP2-4-4-1: Detection and switching control mechanism

DP2-4-4-2: Searching speed control circuitry

FR2-5-1: Capable of detection

FR2-5-1-1: Enhance detection signal

FR2-5-1-2: Avoid false detection

FR2-5-2: Test for the outcome of the bonding process

FR2-5-2-1: Detect non-stick on pad

FR2-5-2-2: Detect non-stick on lead

FR2-5-2-3: Detect the tail left on the capillary after wire-cycle bonding

FR2-5-2-4: Detect ball formation by electronic firing

DP2-5-1: Effective detector

DP2-5-1-1: High-impedance devices

DP2-5-1-2: Noise eliminator

DP2-5-2: Electrically detectable interface system

DP2-5-2-1: Current from bond pad to machine chassis ground

DP2-5-2-2: Current from lead to machine chassis ground

DP2-5-2-3: Current from wire tail to machine chassis ground

DP2-5-2-4: Current from wire tail to wand

FR2-6-1: Support precision machine components with rotational motion

FR2-6-1-1: Robust bearing

FR2-6-1-2: Infinite lifetime/service time

FR2-6-2: Provide motion accuracy of bond head

FR2-6-2-1: Minimize the restoring force

FR2-6-2-2: Increase bonding speed and line pitches

DP2-6-1: Robust linkage with flexure pivot

DP2-6-1-1: Stainless steel cross-flexure pivot bearings

DP2-6-1-2: Zero wear and friction operation

DP2-6-2: Bond head inertia and stiffness in assembly

DP2-6-2-1: Flexure bearing pivots in loading angle

DP2-6-2-2: High stiffness in assembly

3) Vision system

FR3-1-1: Sufficient illumination

FR3-1-2: Clear image features

FR3-2-1: High accuracy and robustness

FR3-2-2: Faster image processing

FR3-3-1: Show bond height difference and capture wider image of bonds

FR3-3-2: Image features requirements

DP3-1-1: Illumination assembly and work light

DP3-1-2: Image acquisition system

DP3-2-1: Pattern matching by image processing tools

DP3-2-2: High-speed processor

DP3-3-1: Mechanism for different magnifications

DP3-3-2: Installation and calibration

4) Material Handling System

FR4-1-1: Pull the materials from input magazine into work holder

FR4-1-2: Support the materials with suitable rail width 
FR4-1-3: Index the materials into bond site and index motion

FR4-1-4: Eject the materials into output magazine

FR4-2-1: Mechanism secures the carrier during bonding operation

FR4-2-2: Hold the fine-pitch and specific materials bonding more stable

FR4-3-1: Raise temperature before entering the bond site FR4-3-2: Maintain the bonding temperature

FR4-3-3: Prevent damage to devices after bonding by rapid heating and cooling

FR4-4: Prevent materials damaged during transportation

FR4-4-1: Prevent devices damaged during transportation from input magazine or into output magazine

FR4-4-2: Prevent devices damaged during injection

FR4-4-3: Prevent devices damaged during ejection

FR4-5-1: Inject/eject the magazine

FR4-5-2: Jog/reverse magazine

DP4-1-1: Injector assembly

DP4-1-2: Front and real rails assembly

DP4-1-3: Indexer assembly

DP4-1-4: Ejector assembly

DP4-2-1: Clamp mechanism

DP4-2-2: Hold down mechanism

DP4-3-1: Pre-heat plate

DP4-3-2: Bond-site heat plate

DP4-3-3: Post-heat plates

DP4-4-1: Wall sensors on both sides of input and output

DP4-4-2: Inject Y-axis sensor detection

DP4-4-3: Jam detection sensor during ejection

DP4-5-1: Gripper motor injects and ejects magazines

DP4-5-2: Z-axis motor drives the jog/reverse motion

5) XY-Table Mechanism

FR5-1-2: Less structural vibration

FR5-2-1: High motion precision

FR5-2-2: High precision in fabrication

DP5-1-1: High acceleration

DP5-1-2: High damping in material and servo loop

DP5-2-1: Decrease joint clearance

DP5-2-2: Decrease the wear of motion joint

\section{REFERENCES}

[1] G. G. Harman, "Wire bonding in microelectronics, material, processes, reliability, and yield," 2nd edition, McGraw Hill, New York, 1997.

[2] C. Boit, R. Weiland, A. Olbrich, U. Muehle, and B. Simmnacher, "Failure analysis concepts for microelectronics technologies and manufacturing of the future," Proceedings of SPIE, Vol. 4406, pp. 1-12, 2001.
[3] D. T. Rooney, D. Nager, D. Geiger, and D. Shanguan, "Evaluation of wire bonding performance, process conditions, and metallurgical integrity of chip on board wire bonds," Microelectronics Reliability, Vol. 45, No. 2, pp. 379-390, 2005.

[4] A. Garg and S. G. Deshmukh, "Maintenance management: Literature review and directions," Journal of Quality Maintenance Engineerings, Vol. 12, No. 3, pp. 205-238, 2006.

[5] M. Murray, K. Fletcher, J. Kennedy, P. Kohler, J. Chambers, and T. Ledwidge, "Capability assurance: A generic model of maintenance," ICOMS-96, Maintenance Engineerings Society of Australia Capability Assurance, paper 72, 1996.

[6] B. S. Blanchard, "System engineering management," John Wiley \& Sons, New York, 1991.

[7] M. Braglia, G. Fantoni, and M. Frosolini, "The house of reliability," International Journal of Quality and Reliability Management, Vol. 24, No. 4, pp. 420-440, 2007.

[8] D. A. Bradley, D. Dawson, N. C. Burd, and A. J. Loader, "Mechatronics: Electronics in products and processes," Chapman \& Hall, London, 1993.

[9] N. P. Suh, "Axiomatic design: Advances and applications," Oxford Press, New York, 2001.

[10] B. S. El-Haik, "Axiomatic quality: Integrating axiomatic design with six-sigma, reliability, and quality Enginee ring," John Wiley \& Sons, New York, 2005.

[11] H. A. Mohsen and E. Cekecek, "Thoughts on the use of axiomatic designs within the product development process," Proceedings of 1st International Conference of Axiomatic Design, Cambridge, Massachusetts, USA, 2000.

[12] G. Taguchi, "Taguchi methods: Signal-to-noise ratio for quality evaluation," Michigan: American Suppliers Institute Press, Vol. 3, 1991.

[13] M. S. Phadke, "Quality engineering using robust design," Prentice Hall, London, 1989.

[14] R. Whitcomb and M. Rioux, "Failure modes and effects analysis (FMEA) system deployment in a semiconductor manufacturing environment," Proceedings IEEE/SEMI Advanced Semiconductor Manufacturing Conference Works, pp. 136-139, 1994.

[15] J. B. Bowles, “An assessment of RPN prioritization in a failure modes effects and criticality analysis," Proceedings Annual Reliability and Maintainability Symposium, pp. 380-386, 2003. 